\title{
Analysis of the Coating Interfacial Stress in Thick Walled Cylinder
}

\author{
Xiangdong Men ${ }^{1,}$, Fenghe Tao ${ }^{1, b}$ and Lin Gan ${ }^{2, c}$ \\ ${ }^{1}$ Mechanical Engineering College, Shijiazhuang 050003, China \\ ${ }^{2}$ PLA University of Science and Technology, Nanjing 210007, China \\ aMenxd1990@163.com, bfhtao63@126.com, cganlin72@163.com
}

Keywords: coating, interfacial stress, thick walled cylinder.

\begin{abstract}
The normal stresses of the coating sprayed on the internal surface of thick walled cylinder, subjected to uniform temperature and pressure change, are determined. The suggested approach enables one to evaluate the normal stresses in the coating and substrate, as well as the interface. The approach is applicable to judge whether high temperature and high pressure fluid will lead to the interface fracture.
\end{abstract}

\section{Introduction}

The stresses arouse in the interface of double layered structures submitted to uniform temperature and pressure change, the components of which are welded, soldered, brazed or cemented together, can give rise to the interface fracture. The stresses in bi-metal thermostats were first discussed by E.Suhir (1986) [1,2]. The stresses in the cylinder (or tube) cannot be determined, without the thick walled cylinder theory. The analysis below contains an engineering theory of normal stresses arising in the coating submitted to uniform temperature and pressure change. The suggested approach enables one to evaluate the normal stresses on the radial, circumferential and axial directions, as well as the magnitude and distribution along the radial direction. Especially, the normal stress along the interface which affects the adhesion is determined by this approach.

\section{Analysis}

Let a kind of coating with uniform thickness, be sprayed onto the internal surface of a thick walled cylinder, the interfacial diameter, outside diameter and inside diameter of which are $a, b$, and $c$ respectively. It is assumed that the boundary conditions are uniform $P_{1}$ on the internal surface and uniform $P_{2}$ on the outside surface, as well as the temperature distribution submitted to the function $T$. (see Fig.1)

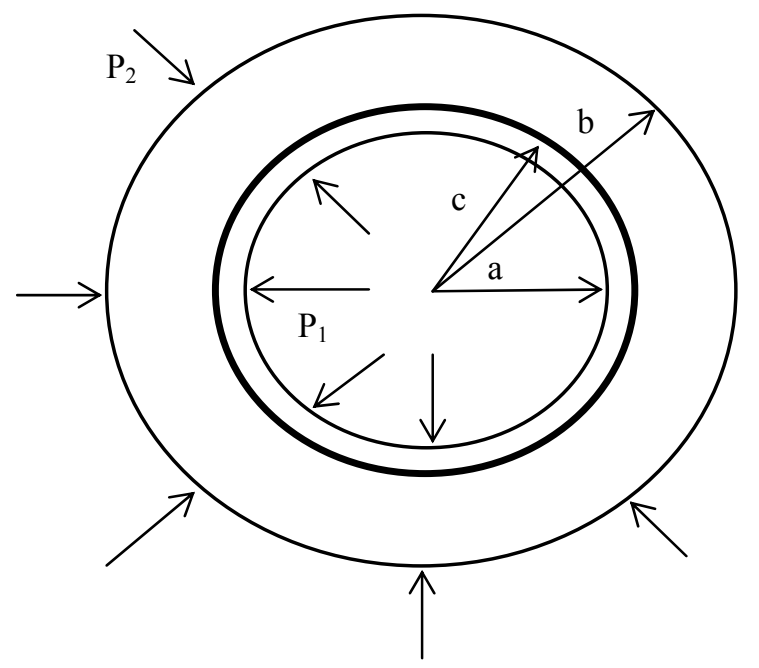

Fig.1 Thick walled cylinder with coating

Based on the thick walled cylinder theory [3], the normal stress components on the radial, circumferential and axial directions are as follows: 


$$
\begin{aligned}
& \sigma_{r}=-\frac{\alpha E}{1-v} \frac{1}{r^{2}} \int_{a}^{r} T \rho d \rho+D_{1}+\frac{D_{2}}{r^{2}} \\
& \sigma_{\theta}=\frac{\alpha E}{1-v}\left[-T+\frac{1}{r^{2}} \int_{a}^{r} T \rho d \rho\right]+D_{1}-\frac{D_{2}}{r^{2}} \\
& \sigma_{z}=-\frac{\alpha E}{1-v} T+2 v D_{1}
\end{aligned}
$$

Where $E$ and $v$, are the elastic constants of the material and $\alpha$ is the thermal expansion for the material, $D_{1}$ and $D_{2}$ are the integration constants depending on the boundary conditions.

$$
\begin{aligned}
& \varepsilon_{r}=\frac{1-v^{2}}{E}\left(\sigma_{r}-\frac{v}{1-v} \sigma_{\theta}\right)+(1+v) \alpha T \\
& \varepsilon_{\theta}=\frac{1-v^{2}}{E}\left(\sigma_{\theta}-\frac{v}{1-v} \sigma_{r}\right)+(1+v) \alpha T
\end{aligned}
$$

is the constitutive equation. Using the displacement formula $u=r \varepsilon_{\theta}$ the longitudinal displacements $u_{c}$ and $u_{s}$ of the coating and substrate are established as follows:

$$
\begin{aligned}
& u_{s}=\frac{1-v_{s}^{2}}{c E_{s}}\left[c^{2}\left(\frac{1-2 v_{s}}{1-v_{s}}\right) \sigma_{r 0}-\frac{2 c^{2} b^{2}}{b^{2}-c^{2}}\left(\sigma_{r 0}-P_{2}-\frac{\alpha_{s} E_{s}}{1-v_{s}} \frac{1}{b^{2}} \int_{c}^{b} T \rho d \rho\right)\right] \\
& u_{c}=\frac{1-v_{c}^{2}}{c E_{c}}\left[\frac{\alpha_{c} E_{c}}{\left(1-v_{c}\right)^{2}} \int_{a}^{c} T \rho d \rho+c^{2}\left(\frac{1-2 v_{c}}{1-v_{c}}\right) P_{1}-\frac{c^{2}\left(c^{2}+a^{2}\right)-c^{2} \frac{v_{c}}{1-v_{c}}\left(c^{2}-a^{2}\right)}{c^{2}-a^{2}}\left(P_{1}-\sigma_{r 0}-\frac{\alpha_{c} E_{c}}{1-v_{c}} \frac{1}{c^{2}} \int_{a}^{c} T \rho d \rho\right)\right]
\end{aligned}
$$

where $E_{c}, v_{c}$ and $E_{s}, v_{s}$ are the elastic constants of coating and substrate, $\alpha_{c}$ and $\alpha_{s}$ are the thermal expansion for coating and substrate, $\sigma_{r 0}$ is the normal stress on the radial direction of the interface.

We assume that $T_{c}=\frac{\alpha_{c} E_{c}}{1-v_{c}} \int_{a}^{c} T \rho d \rho, T_{s}=\frac{\alpha_{s} E_{s}}{1-v_{s}} \int_{c}^{b} T \rho d \rho$ are the thermal expansion stress coefficients, and $D_{c}=\frac{c^{2}+a^{2}}{c^{2}-a^{2}}, D_{s}=\frac{2 c^{2}}{b^{2}-c^{2}}$ are the dimension coefficients, so that the equation can be simplified to

$$
\begin{aligned}
& u_{s}=\frac{1-v_{s}^{2}}{c E_{s}}\left[c^{2}\left(\frac{1-2 v_{s}}{1-v_{s}}\right) \sigma_{r 0}-b^{2} D_{s}\left(\sigma_{r 0}-P_{2}-\frac{1}{b^{2}} T_{s}\right)\right] \\
& u_{c}=\frac{1-v_{c}^{2}}{c E_{c}}\left[\frac{1}{\left(1-v_{c}\right)} T_{c}+c^{2}\left(\frac{1-2 v_{c}}{1-v_{c}}\right) P_{1}-c^{2}\left(D_{c}-\frac{v_{c}}{1-v_{c}}\right)\left(P_{1}-\sigma_{r 0}-\frac{1}{c^{2}} T_{c}\right)\right]
\end{aligned}
$$

Using the condition $u_{s}=u_{c}$ of the displacement compatibility, we have:

$$
\sigma_{r 0}=\frac{\left(1+D_{c}\right) T_{c}+c^{2}\left(1-D_{c}\right) P_{1}-E^{*} b^{2} D_{s} P_{2}-E^{*} D_{s} T_{s}}{\left\{E^{*}\left[c^{2}\left(\frac{1-2 v_{s}}{1-v_{s}}\right)-b^{2} D_{s}\right]-c^{2}\left(D_{c}-\frac{v_{c}}{1-v_{c}}\right)\right\}}
$$

Where $E^{*}=\frac{E_{c}}{E_{s}}\left(\frac{1-v_{s}^{2}}{1-v_{c}^{2}}\right)$ is the relative elastic constant?

We assume that $K$ subjects $K=E^{*}\left[c^{2}\left(\frac{1-2 v_{s}}{1-v_{s}}\right)-b^{2} D_{s}\right]-c^{2}\left(D_{c}-\frac{v_{c}}{1-v_{c}}\right)$ and $A_{1}=\frac{\left(1+D_{c}\right)}{K}, A_{2}=\frac{E^{*} D_{s}}{K}$, $B_{1}=\frac{c^{2}\left(1-D_{c}\right)}{K}, B_{2}=\frac{E^{*} b^{2} D_{s}}{K}$ are the weighting factors. Finally we get the expression:

$$
\sigma_{r 0}=A_{1} T_{c}-A_{2} T_{s}+B_{1} P_{1}-B_{2} P_{2}
$$

The formula above visually indicates the relationship between $\sigma_{r 0}$ and $T_{c}, T_{s}, P_{1}, P_{2}$. Generally, to a system, $A_{1}, A_{2}$ and $B_{1}, B_{2}$ are the inherent parameters, which characterize the influence to $\sigma_{r 0}$.

Similarly, the $\sigma_{\theta 0}$ and $\sigma_{z 0}$ can be determined. For coating interfacial problem, only normal stress on the radial direction is considered. The $\sigma_{r}$ and $\sigma_{\theta}$ of coating and substrate can be respectively determined with $\sigma_{r 0}$ :

$$
\sigma_{r c}=\left[-\frac{1}{r^{2}} \frac{\alpha_{c} E_{c}}{1-v_{c}} \int_{a}^{r} T \rho d \rho+\frac{a^{2}\left(c^{2}-r^{2}\right)}{r^{2}\left(c^{2}-a^{2}\right)} P_{1}+\frac{c^{2}\left(r^{2}-a^{2}\right)}{r^{2}\left(c^{2}-a^{2}\right)} \sigma_{r 0}+\frac{\left(r^{2}-a^{2}\right)}{r^{2}\left(c^{2}-a^{2}\right)} T_{c}\right]
$$




$$
\begin{aligned}
& \sigma_{\theta c}=\left[-\frac{\alpha_{c} E_{c}}{1-v_{c}} T+\frac{1}{r^{2}} \frac{\alpha_{c} E_{c}}{1-v_{c}} \int_{a}^{r} T \rho d \rho-\frac{a^{2}\left(r^{2}+c^{2}\right)}{r^{2}\left(c^{2}-a^{2}\right)} P_{1}+\frac{c^{2}\left(r^{2}+a^{2}\right)}{r^{2}\left(c^{2}-a^{2}\right)} \sigma_{r 0}+\frac{\left(r^{2}+a^{2}\right)}{r^{2}\left(c^{2}-a^{2}\right)} T_{c}\right] \\
& \sigma_{r s}=\left[-\frac{1}{r^{2}} \frac{\alpha_{s} E_{s}}{1-v_{s}} \int_{c}^{r} T \rho d \rho+\frac{c^{2}\left(b^{2}-r^{2}\right)}{r^{2}\left(b^{2}-c^{2}\right)} \sigma_{r 0}+\frac{b^{2}\left(r^{2}-c^{2}\right)}{r^{2}\left(b^{2}-c^{2}\right)} P_{2}+\frac{\left(r^{2}-c^{2}\right)}{r^{2}\left(b^{2}-c^{2}\right)} T_{s}\right] \\
& \sigma_{\theta s}=\left[-\frac{\alpha_{s} E_{s}}{1-v_{s}} T+\frac{1}{r^{2}} \frac{\alpha_{s} E_{s}}{1-v_{s}} \int_{c}^{r} T \rho d \rho-\frac{c^{2}\left(r^{2}+b^{2}\right)}{r^{2}\left(b^{2}-c^{2}\right)} \sigma_{r 0}+\frac{b^{2}\left(r^{2}+c^{2}\right)}{r^{2}\left(b^{2}-c^{2}\right)} P_{2}+\frac{\left(r^{2}+c^{2}\right)}{r^{2}\left(b^{2}-c^{2}\right)} T_{s}\right]
\end{aligned}
$$

\section{Numerical Example}

The following numerical example is executed for $\mathrm{NiCr}-\mathrm{Cr}_{3} \mathrm{C}_{2}$ coatings spraying onto the inside surface of $40 \mathrm{CrMn}$ cylinder. After high temperature heat flux flows through the cylinder, the average temperature of internal coating area reaches $1100 \mathrm{~K}$, and the average temperature of substrate average is $300 \mathrm{~K}$. The pressures inside and outside the cylinder are neglected. The structure parameters are $a=49.8 \mathrm{~mm}, b=70 \mathrm{~mm}, c=50 \mathrm{~mm}$, The material parameters are as follows:

Table 1 Material performance parameters of the coating and substrate

\begin{tabular}{ccccc}
\hline \multirow{2}{*}{ Materials } & Elasticity Modulus & $\begin{array}{c}\text { Coefficient of } \\
\text { Linear Expansion }\end{array}$ & Poisson's Ratio & Thermal Conductivity \\
\cline { 2 - 5 } & $E / \mathrm{MPa}$ & $\alpha / 10^{-6} \mathrm{~K}$ & $v$ & $\lambda / 10^{-6}$ \\
\hline Substrate & $165 \times 10^{3}$ & 14 & 0.3 & 8.9 \\
Coating & $160 \times 10^{3}$ & 11.5 & 024 & 4.06 \\
\hline
\end{tabular}

The calculated stresses of coating and substrate are plotted in Fig. 2 and Fig.3. The normal stress of the interface is $\sigma_{r 0}=-6.7 \mathrm{MPa}$. The minus sign indicates that the force is on the contrary of the material outward normal direction, i.e., the stress state is compressive.
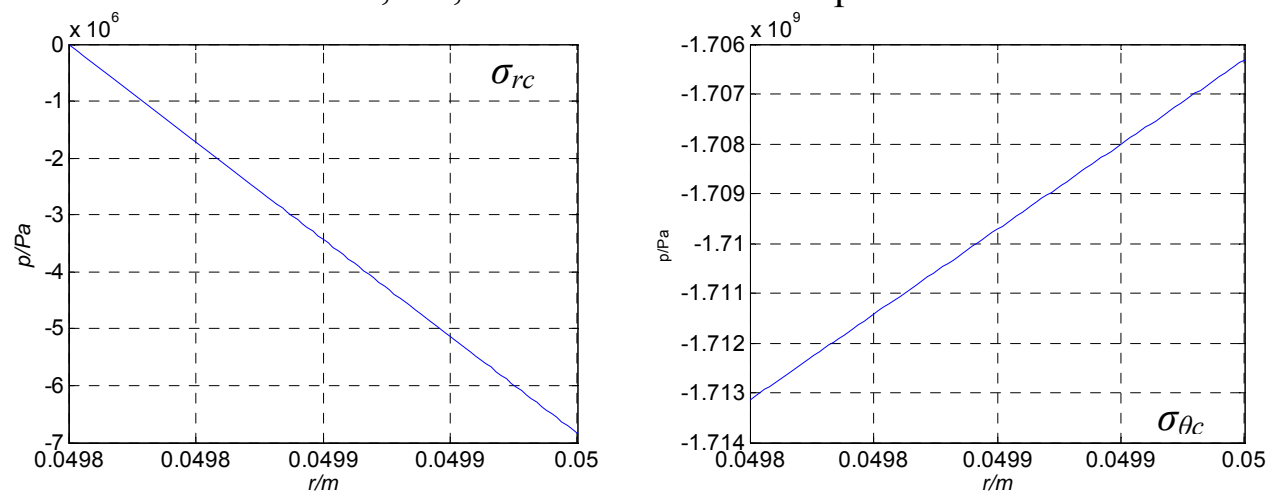

Fig.2 Calculated Stress of Coating
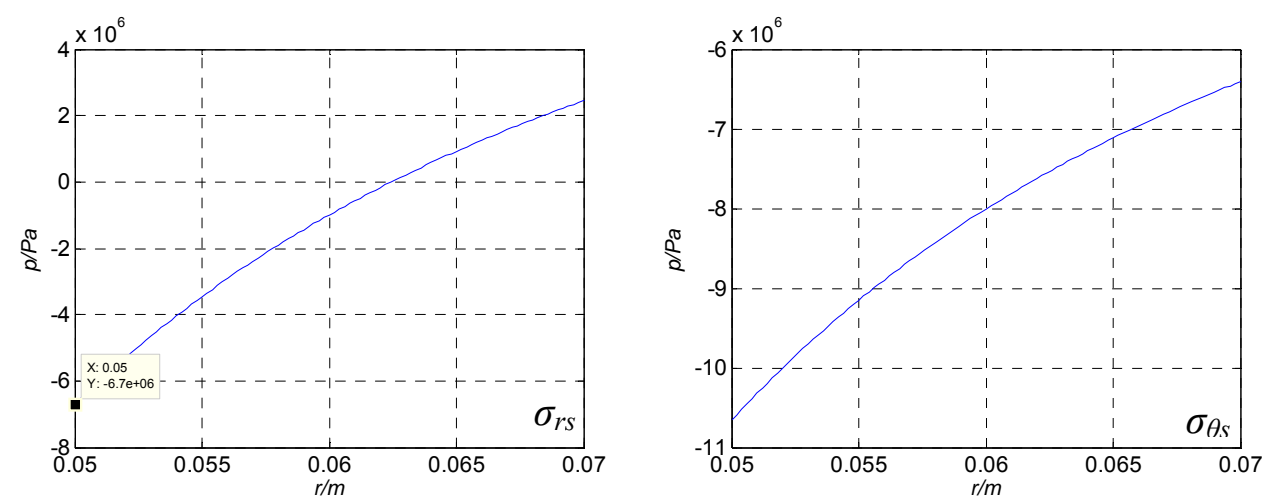

Fig.3 Calculated Stress of Substrate 


\section{Discussion}

We now consider how the results of the above analysis might help our understanding of the stresses in composite structures. Due to the virtues of axisymmetric structure, the stress distribution is relatively simple, with no shear and moment in the structure. The normal stresses acting in the coating and cylinder, especially in the interface, are determined. While the normal stresses, of different directions, in the coating are responsible for the strength of the film materials, the interfacial stress is responsible for blistering and peeling.

Practically, the temperature and pressure of the structure always change with time. Thus, the stresses should be calculated according to the temporal sequence, and the stress state should be judged by the sign. Generally, special attention should be paid to the magnitude and state of the interfacial stress.

\section{Summary}

An engineering theory of stresses in the coating sprayed on the inside surface of thick wall cylinder is put forward. The obtained formulae are simple, easy-to-use, and clearly indicate the role of the major factors affecting the stresses. The results of analysis can be utilized for guidance in the physical design and detection of coating and cylinder structures.

\section{References}

[1] E.Suhir. Stresses in Bi-Metal Thermostats. Journal of Applied Mechanics. Vol. 53 (1986) No. 9, p. 657-660.

[2] E.Suhir. an Approximate Analysis of stresses in Multilayered Elastic Thin Films. Journal of Applied Mechanics. Vol. 55 (1988) No. 3, p. 143-148.

[3] Seifi, Rahman. Stress intensity factors for internal surface cracks in autofrettaged functionally graded thick cylinders using weight function method. Theoretical and Applied Fracture Mechanics. Vol. 75 (2015) p. 113-123. 